\title{
Page S-1
}

\section{A Chemical Activation Study of the Unimolecular Reactions of $\mathrm{CD}_{3} \mathrm{CD}_{2} \mathrm{CHCl}_{2}$ and $\mathrm{CHCl}_{2} \mathrm{CHCl}_{2}$ with} Analysis of the 1,1-HCl Elimination Pathway.

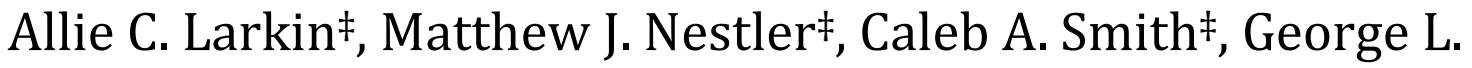 \\ Heard ${ }^{\ddagger}$ D. W. Setser ${ }^{\dagger}$ and Bert E. Holmes ${ }^{*}$ \\ ‡ Department of Chemistry, University of N. Carolina-Asheville, One University \\ Heights, Asheville, North Carolina 28804-8511 and † Department of Chemistry, \\ Kansas State University, Manhattan, Kansas, 66506.
}




\section{Page S-2}

Supplemental Data Glossary

3-6: Calculated Frequencies and Moments of Inertia

7-12: Cartesian Coordinates of Relevant Species

12: Absolute and Zero Point Energies of Relevant Species

13: $\mathrm{CH}_{3} \mathrm{CHCl}_{2}$ Gaussian Graphic

14-18: Calculated Rate Constants

19-23: Experimental Data 


\section{Page S-3 Table S1: Calculated frequencies and}

moments of inertia for $\mathrm{CHCl}_{2} \mathrm{CHCl}_{2}$ at $\mathrm{MP2} / 6-311+\mathrm{G}(2 \mathrm{~d}, \mathrm{p})$

\begin{tabular}{|c|c|c|c|}
\hline \multirow{19}{*}{$\begin{array}{l}\text { Frequencies } \\
\qquad\left(\mathrm{cm}^{-1}\right)\end{array}$} & Ground State & $1,1 \mathrm{HCl} \mathrm{TS}$ & 1,2-HCl TS \\
\hline & 60.93 & --- & --- \\
\hline & 171.93 & 35.81 & 68.02 \\
\hline & 231.33 & 50.59 & 129.66 \\
\hline & 300.56 & 106.72 & 176.85 \\
\hline & 303.17 & 186.41 & 204.34 \\
\hline & 320.16 & 268.22 & 269.20 \\
\hline & 371.89 & 308.97 & 355.22 \\
\hline & 729.22 & 546.47 & 405.09 \\
\hline & 775.42 & 662.13 & 480.34 \\
\hline & 788.17 & 733.86 & 641.02 \\
\hline & 831.80 & 836.79 & 854.58 \\
\hline & 1081.89 & 910.65 & 883.11 \\
\hline & 1217.31 & 984.06 & 940.20 \\
\hline & 1240.66 & 1148.61 & 1124.45 \\
\hline & 1244.84 & 1173.37 & 1269.12 \\
\hline & 1347.09 & 1237.74 & 1383.93 \\
\hline & 3165.03 & 1312.20 & 1562.85 \\
\hline & 3177.67 & 3183.67 & 3257.11 \\
\hline $\mathrm{I}_{\mathrm{x}}\left(\mathrm{amu} \AA^{2}\right)$ & 304.45 & 337.78 & 361.49 \\
\hline $\mathrm{I}_{\mathrm{y}}$ & 378.08 & 632.45 & 453.67 \\
\hline $\mathrm{I}_{\mathrm{z}}$ & 666.88 & 682.24 & 619.77 \\
\hline Ired $\left(\operatorname{amu} \AA^{2}\right)$ & 79.11 & 99.69 & --- \\
\hline
\end{tabular}




\section{Page S-4}

\section{Table S2: Calculated frequencies and moments of inertia for $\mathrm{CD}_{2} \mathrm{ClCHCl}_{2}$ at MP2/6-311+G(2d,p)}

\begin{tabular}{|c|c|c|c|c|}
\hline & Ground State & $1,1 \mathrm{HCl} \mathrm{TS}$ & 2,1-DCl TS & 1,2-HCl TS \\
\hline & 109.80 & -- & -- & --- \\
\hline & 175.45 & 40.29 & 103.75 & 84.12 \\
\hline & 256.33 & 61.76 & 134.03 & 160.72 \\
\hline & 333.77 & 131.20 & 177.66 & 292.15 \\
\hline & 357.36 & 271.40 & 300.52 & 306.48 \\
\hline & 637.60 & 331.54 & 445.62 & 363.71 \\
\hline & 737.65 & 528.25 & 527.08 & 391.32 \\
\hline & 770.40 & 757.44 & 609.20 & 573.42 \\
\hline & 804.99 & 800.08 & 708.38 & 585.34 \\
\hline Froguencioc & 921.02 & 846.07 & 803.29 & 703.31 \\
\hline$\left(\mathrm{cm}^{-1}\right)$ & 972.70 & 890.74 & 871.38 & 813.21 \\
\hline & 1060.44 & 923.89 & 901.54 & 972.70 \\
\hline & 1155.84 & 959.04 & 995.95 & 1061.10 \\
\hline & 1257.41 & 1022.35 & 1111.48 & 1094.83 \\
\hline & 1303.28 & 1154.80 & 1284.47 & 1326.73 \\
\hline & 2272.35 & 1411.93 & 1456.33 & 1495.36 \\
\hline & 2379.01 & 2212.49 & 2337.09 & 2327.60 \\
\hline & 3178.08 & 2322.69 & 3257.60 & 2479.05 \\
\hline $\mathrm{I}_{\mathrm{x}}\left(\mathrm{amu} \AA^{2}\right)$ & 149.80 & 288.30 & 206.42 & 191.11 \\
\hline $\mathrm{I}_{\mathrm{y}}$ & 346.90 & 348.99 & 329.68 & 364.43 \\
\hline $\mathrm{I}_{\mathrm{z}}$ & 475.97 & 616.83 & 477.61 & 488.25 \\
\hline $\mathrm{I}_{\text {red }}\left(\mathrm{amu} \AA^{2}\right)$ & 32.63 & 39.39 & --- & --- \\
\hline
\end{tabular}


Page S-5

Table S3: Calculated frequencies and moments of inertia for $\mathrm{CD}_{3} \mathrm{CD}_{2} \mathrm{CHCl}_{2}$ at MP2/6-311G(2d,p)

\begin{tabular}{|c|c|c|c|}
\hline \multirow{19}{*}{$\begin{array}{l}\text { Frequencies } \\
\qquad\left(\mathrm{cm}^{-1}\right)\end{array}$} & $\begin{array}{l}\text { Ground } \\
\text { State }\end{array}$ & $1,1 \mathrm{HCl} \mathrm{TS}$ & 1,2-DCl TS \\
\hline & 97.07 & --- & --- \\
\hline & 175.59 & 45.62 & 100.78 \\
\hline & 196.56 & 50.18 & 132.69 \\
\hline & 270.06 & 73.24 & 144.79 \\
\hline & 348.01 & 166.03 & 237.09 \\
\hline & 370.58 & 301.53 & 304.88 \\
\hline & 576.67 & 373.85 & 377.62 \\
\hline & 658.87 & 535.90 & 423.05 \\
\hline & 721.15 & 666.70 & 598.92 \\
\hline & 811.86 & 732.67 & 720.80 \\
\hline & 902.48 & 884.01 & 836.03 \\
\hline & 930.85 & 902.72 & 871.70 \\
\hline & 982.42 & 958.61 & 886.13 \\
\hline & 997.31 & 984.16 & 935.02 \\
\hline & 1089.00 & 1013.62 & 965.46 \\
\hline & 1098.40 & 1036.30 & 1026.14 \\
\hline & 1102.51 & 1050.35 & 1091.92 \\
\hline & 1158.29 & 1098.99 & 1099.29 \\
\hline
\end{tabular}




\begin{tabular}{|c|c|c|c|}
\hline & 1213.34 & 1101.48 & 1148.54 \\
\hline & 1271.38 & 1145.11 & 1172.86 \\
\hline & 1307.13 & 1167.53 & 1291.76 \\
\hline & 2211.03 & 1262.63 & 1509.00 \\
\hline & 2245.42 & 2156.58 & 2211.82 \\
\hline & 2327.50 & 2216.93 & 2344.84 \\
\hline & 2339.47 & 2290.93 & 2348.89 \\
\hline & 2353.13 & 2354.94 & 2354.33 \\
\hline & 3163.18 & 2361.00 & 3253.53 \\
\hline $\mathrm{I}_{\mathrm{x}}\left(\mathrm{amu} \AA^{2}\right)$ & 150.08 & 220.55 & 210.28 \\
\hline $\mathrm{I}_{\mathrm{y}}$ & 245.26 & 340.98 & 246.41 \\
\hline $\mathrm{I}_{\mathrm{z}}$ & 370.48 & 539.06 & 427.18 \\
\hline Ired $\left(\right.$ amu $\AA^{2}$ ) & 27.05 & 28.50 & --- \\
\hline
\end{tabular}

\section{Page S-6}




\section{Page S-7}

Gaussian Cartesian Coordinates for Ground State and Transition State Geometries of $\mathrm{CHCl}_{2} \mathrm{CHCl}_{2}, \mathrm{CD}_{2} \mathrm{ClCHCl}_{2}$, and $\mathrm{CD}_{3} \mathrm{CD}_{2} \mathrm{CHCl}_{2}$ all at MP2/6-311G(2d,p)

Coordinates unformatted for ease of transfer

\section{List S-1 Coordinates of $\mathrm{CHCl}_{2} \mathrm{CHCl}_{2}$ Initial Ground State}

$\begin{array}{llrrrc}\text { Number } & \text { Atomic Number } & \text { Atomic Type } & \mathrm{X} & \mathrm{Y} & \mathrm{Z} \\ 1 & 6 & 0 & -0.663067 & -0.000149 & -0.374673 \\ 2 & 1 & 0 & -0.495593 & -0.000104 & -1.449164 \\ 3 & 6 & 0 & 0.663026 & -0.000101 & 0.374686 \\ 4 & 1 & 0 & 0.495670 & -0.000100 & 1.449179 \\ 5 & 17 & 0 & -1.602495 & 1.456235 & 0.040224 \\ 6 & 17 & 0 & -1.602466 & -1.456227 & 0.040854 \\ 7 & 17 & 0 & 1.602451 & 1.456283 & -0.040228 \\ 8 & 17 & 0 & 1.602521 & -1.456191 & -0.040856\end{array}$

\section{List S-2 Coordinates of $\mathrm{CHCl}_{2} \mathrm{CHCl}_{2} \mathbf{1 , 1} \mathbf{H C l}$ Elimination Transition State}

$\begin{array}{lccrrrr}\text { Number } & \text { Atomic Number } & \text { Atomic Type } & X & Y & Z \\ 1 & 6 & 0 & 0.551057 & -0.558430 & 0.370542 \\ 2 & 1 & 0 & 0.027238 & -1.336251 & 0.919746 \\ 3 & 17 & 0 & 1.696577 & 0.242984 & 1.443410 \\ 4 & 17 & 0 & 1.339493 & -1.346705 & -1.059317 \\ 5 & 6 & 0 & -0.396173 & 0.221599 & -0.464491 \\ 6 & 17 & 0 & 0.021568 & 1.803489 & -0.780193 \\ 7 & 17 & 0 & -3.007052 & -0.488935 & 0.394859 \\ 8 & 1 & 0 & -1.816507 & -0.226918 & -0.334950\end{array}$




\section{Page S-8}

\section{List S-3 Coordinates of $\mathrm{CHCl}_{2} \mathrm{CHCl}_{2} \mathbf{1 , 2} \mathrm{HCl}$ Elimination Transition State}

$\begin{array}{rccrrrr}\text { Number } & \text { Atomic Number } & \text { Atomic Type } & \text { X } & Y \\ 1 & 6 & 0 & -0.646711 & 0.048704 & 0.024063 \\ 2 & 1 & 0 & 0.067341 & 0.857028 & 0.678716 \\ 3 & 6 & 0 & 0.505445 & -0.142767 & -0.778590 \\ 4 & 1 & 0 & 0.704820 & 0.533833 & -1.599282 \\ 5 & 17 & 0 & 1.608760 & 1.659675 & 0.649632 \\ 6 & 17 & 0 & 1.504266 & -1.475847 & -0.648642 \\ 7 & 17 & 0 & -1.199776 & -1.244774 & 1.055565 \\ 8 & 17 & 0 & -1.908812 & 1.012329 & -0.736100\end{array}$

\section{List S-4 Coordinates of $\mathrm{CD}_{2} \mathrm{ClCHCl}_{2}$ Initial Ground State}

Number Atomic Number Atomic Type

$\begin{array}{ccc}X & Y & Z \\ 0.411705 & -0.080465 & -0.365732 \\ 0.261023 & -0.135005 & -1.440231 \\ 0.424477 & 1.642105 & 0.081660 \\ -0.646974 & -0.847189 & 0.389764 \\ -0.563116 & -1.905182 & 0.149013 \\ -0.533976 & -0.697808 & 1.461866 \\ 1.994234 & -0.840870 & -0.011356 \\ -2.286494 & -0.312769 & -0.088824\end{array}$




\section{Page $S-9$}

\section{List S-5 Coordinates of $\mathrm{CD}_{2} \mathrm{ClCHCl}_{2} \mathrm{1,1} \mathbf{H C l}$ Elimination Transition State}

$\begin{array}{cccccc}\text { Number } & \text { Atomic Number } & \text { Atomic Type } & \mathrm{X} & \mathrm{Y} & \mathrm{Z} \\ 1 & 6 & 0 & 0.143907 & 0.564964 & -0.309814 \\ 2 & 1 & 0 & 0.928716 & -0.755189 & -0.464172 \\ 3 & 17 & 0 & 1.661933 & -1.827167 & 0.052344 \\ 4 & 17 & 0 & 1.043572 & 1.965854 & -0.062338 \\ 5 & 6 & 0 & -1.103214 & 0.576859 & 0.482737 \\ 6 & 1 & 0 & -1.573733 & 1.558546 & 0.555938 \\ 7 & 1 & 0 & -0.802744 & 0.261472 & 1.492042 \\ 8 & 17 & 0 & -2.281764 & -0.604321 & -0.144203\end{array}$

\section{List S-6 Coordinates of $\mathrm{CD}_{2} \mathrm{ClCHCl}_{2} 2,1 \mathrm{DCl}$ Elimination Transition State}

$\begin{array}{cccccc}\text { Number } & \text { Atomic Number } & \text { Atomic Type } & \mathrm{X} & \mathrm{Y} & \mathrm{Z} \\ 1 & 6 & 0 & -0.539424 & -0.716987 & 0.735210 \\ 2 & 1 & 0 & 0.477760 & -1.192282 & 0.205494 \\ 3 & 6 & 0 & 0.168294 & 0.500463 & 0.802118 \\ 4 & 1 & 0 & 0.891490 & 0.660038 & 1.590946 \\ 5 & 17 & 0 & 2.157624 & -0.764514 & -0.255042 \\ 6 & 17 & 0 & -0.133793 & 1.812337 & -0.187171 \\ 7 & 17 & 0 & -1.936385 & -0.866885 & -0.305011 \\ 8 & 1 & 0 & -0.629043 & -1.244564 & 1.682387\end{array}$




\section{Page S-10}

\section{List S-7 Coordinates of $\mathrm{CD}_{2} \mathrm{ClCHCl}_{2} \mathbf{1 , 2} \mathrm{HCl}$ Elimination Transition State}

$\begin{array}{cccccc}\text { Number } & \text { Atomic Number } & \text { Atomic Type } & \mathrm{X} & \mathrm{Y} & \mathrm{Z} \\ 1 & 6 & 0 & -0.184318 & 0.460224 & 0.000000 \\ 2 & 1 & 0 & 0.415144 & -0.696320 & 0.000000 \\ 3 & 6 & 0 & -1.205012 & -0.492411 & 0.000000 \\ 4 & 17 & 0 & 0.220299 & -2.426739 & 0.000000 \\ 5 & 17 & 0 & 0.220299 & 1.286768 & 1.483728 \\ 6 & 1 & 0 & -1.657205 & -0.803053 & -0.932013 \\ 7 & 1 & 0 & -1.657205 & -0.803053 & 0.932013 \\ 8 & 17 & 0 & 0.220299 & 1.286768 & -1.483728\end{array}$

\section{List S-8 Coordinates of $\mathrm{CD}_{3} \mathrm{CD}_{2} \mathrm{CHCl}_{2}$ Initial Ground State}

$\begin{array}{cccccc}\text { Number } & \text { Atomic Number } & \text { Atomic Type } & \mathrm{X} & \mathrm{Y} & \mathrm{Z} \\ 1 & 6 & 0 & -0.899950 & -1.014760 & -0.316248 \\ 2 & 1 & 0 & -0.773730 & -0.911960 & -1.396655 \\ 3 & 1 & 0 & -0.644720 & -2.045451 & -0.054610 \\ 4 & 6 & 0 & -2.333882 & -0.706901 & 0.102487 \\ 5 & 1 & 0 & -2.447690 & -0.775155 & 1.187095 \\ 6 & 1 & 0 & -3.017677 & -1.425564 & -0.351393 \\ 8 & 6 & 0 & 0.109556 & -0.126034 & 0.372986 \\ 9 & 1 & 0 & 0.021557 & -0.169778 & 1.455930 \\ 10 & 17 & 0 & 1.779196 & -0.646268 & -0.017377 \\ 11 & 17 & 0 & -0.117921 & 1.594568 & -0.075883\end{array}$




\section{Page S-11}

\section{List S-9 Coordinates of $\mathrm{CD}_{3} \mathrm{CD}_{2} \mathrm{CHCl}_{2} 1,1 \mathrm{HCl}$ Elimination Transition State}

$\begin{array}{cccccc}\text { Number } & \text { Atomic Number } & \text { Atomic Type } & \mathrm{X} & \mathrm{Y} & \mathrm{Z} \\ 1 & 6 & 0 & 0.505125 & -0.095205 & -0.010233 \\ 2 & 1 & 0 & -1.033287 & -0.295844 & -0.077714 \\ 3 & 17 & 0 & -2.448652 & -0.399892 & 0.023987 \\ 4 & 17 & 0 & 1.550981 & -1.410279 & -0.087098 \\ 5 & 6 & 0 & 1.122464 & 1.169962 & 0.389580 \\ 6 & 1 & 0 & 0.857163 & 1.167720 & 1.466837 \\ 7 & 1 & 0 & 2.214277 & 1.191822 & 0.327096 \\ 8 & 6 & 0 & 0.439036 & 2.374121 & -0.269678 \\ 9 & 1 & 0 & 0.799059 & 3.298585 & 0.181825 \\ 10 & 1 & 0 & 0.665832 & 2.401885 & -1.335640 \\ 11 & 1 & 0 & -0.642390 & 2.315475 & -0.147530\end{array}$

\section{List S-10 Coordinates of $\mathrm{CD}_{3} \mathrm{CD}_{2} \mathrm{CHCl}_{2}$ 1,2 $\mathrm{DCl}$ Elimination Transition State}

$\begin{array}{cccccc}\text { Number } & \text { Atomic Number } & \text { Atomic Type } & \mathrm{X} & \mathrm{Y} & \mathrm{Z} \\ 1 & 6 & 0 & 0.636492 & -1.047101 & -0.455980 \\ 2 & 1 & 0 & 0.239866 & -1.505308 & -1.358205 \\ 3 & 1 & 0 & 0.870957 & 0.130878 & -0.710516 \\ 4 & 6 & 0 & -0.320168 & -0.502527 & 0.411705 \\ 5 & 1 & 0 & -0.096423 & -0.231510 & 1.435407 \\ 6 & 17 & 0 & 0.695622 & 1.846309 & -0.023291 \\ 7 & 17 & 0 & -1.939436 & -0.334198 & -0.024985 \\ 8 & 6 & 0 & 1.917053 & -1.600461 & 0.142610\end{array}$




\section{Page S-12}

$$
9
$$

10

11

\section{1}

0

0

0
$2.693847-1.645443 \quad-0.620144$

$2.271805 \quad-0.949057 \quad 0.943050$

$\begin{array}{lll}1.764522 & -2.604903 & 0.541082\end{array}$

\section{Table S-4 Calculated Absolute Energies and Zero Point Energies for Ground and Transition State Geometries of $\mathrm{CHCl}_{2} \mathrm{CHCl}_{2}, \mathrm{CD}_{2} \mathrm{ClCHCl}_{2}$, and $\mathrm{CD}_{3} \mathrm{CD}_{2} \mathrm{CHCl}_{2}$ all at MP2/6- $311 G(2 d, p)$}

\begin{tabular}{|l|l|l|}
\hline Geometry & $\begin{array}{l}\text { Absolute Energy } \\
\text { (Hartree/Particle) }\end{array}$ & $\begin{array}{l}\text { Zero Point Energy } \\
\text { Correction } \\
\text { (Hartree/Particle) }\end{array}$ \\
\hline $\mathrm{CHCl}_{2} \mathrm{CHCl}_{2}$ Initial Ground State & -1915.8736435830 & 0.039547 \\
\hline $\mathrm{CHCl}_{2} \mathrm{CHCl}_{2} 1,1 \mathrm{HCl}$ Elimination TS & -1915.7539646776 & 0.031180 \\
\hline $\mathrm{CHCl}_{2} \mathrm{CHCl}_{2} 1,2 \mathrm{HCl}$ Elimination TS & -1915.7646814473 & 0.031906 \\
\hline $\mathrm{CD}_{2} \mathrm{ClCHCl}_{2}$ Initial Ground State & -1456.8065562226 & 0.042564 \\
\hline $\mathrm{CD}_{2} \mathrm{ClCHCl}_{2} 1,1 \mathrm{HCl}$ Elimination TS & -1456.6826013950 & 0.033412 \\
\hline $\mathrm{CD}_{2} \mathrm{ClCHCl}_{2} 2,1 \mathrm{DCl}$ Elimination TS & -1456.7004135638 & 0.036508 \\
\hline $\mathrm{CD}_{2} \mathrm{ClCHCl}_{2} 1,2 \mathrm{HCl}$ Elimination TS & -1456.6898759476 & 0.034244 \\
\hline $\mathrm{CD}_{3} \mathrm{CD}_{2} \mathrm{CHCl}_{2}$ Initial Ground State & -1036.9350004808 & 0.070439 \\
\hline $\mathrm{CD}_{3} \mathrm{CD}_{2} \mathrm{CHCl}_{2} 1,1 \mathrm{HCl}$ Elimination TS & -1036.8180266155 & 0.061355 \\
\hline $\mathrm{CD}_{3} \mathrm{CD}_{2} \mathrm{CHCl}_{2} 1,2 \mathrm{DCl}$ Elimination TS & -1036.8362582455 & 0.064672 \\
\hline
\end{tabular}




\section{Page S-13}

Table S-5 Absolute Energies (all negative) and Zero Point Energies (ZPE) for all theory levels at $6-311+G(2 d, p)$ for all molecules used in Isodesmic Reaction (6) in Hartree/Particle

\begin{tabular}{|c|c|c|c|c|c|c|}
\hline & $\begin{array}{l}\text { MP2 } \\
\text { Absolute }\end{array}$ & $\begin{array}{l}\text { MP2 } \\
\text { ZPE }\end{array}$ & $\begin{array}{l}\text { B3PW91 } \\
\text { Absolute }\end{array}$ & $\begin{array}{l}\text { B3PW91 } \\
\text { ZPE }\end{array}$ & $\begin{array}{l}\text { M062X } \\
\text { Absolute }\end{array}$ & $\begin{array}{l}\text { M062X } \\
\text { ZPE }\end{array}$ \\
\hline $\mathrm{CH}_{3} \mathrm{CH}_{3}$ & $\begin{array}{l}- \\
79.58631 \\
2244205\end{array}$ & $\begin{array}{l}0.0757 \\
16\end{array}$ & $\begin{array}{l}- \\
79.82714 \\
10219\end{array}$ & 0.074414 & $\begin{array}{l}- \\
79.799111 \\
4230\end{array}$ & 0.074705 \\
\hline $\mathrm{CH}_{3} \mathrm{CHCl}_{2}$ & $\begin{array}{l}- \\
997.7359 \\
9264515\end{array}$ & $\begin{array}{l}0.0582 \\
47\end{array}$ & $\begin{array}{l}998.9673 \\
12269\end{array}$ & 0.074414 & $\begin{array}{l}999.00199 \\
1392\end{array}$ & 0.057793 \\
\hline $\mathrm{CH}_{3} \mathrm{CH}_{2} \mathrm{CH}_{3}$ & $\begin{array}{l}- \\
118.7887 \\
9268353\end{array}$ & $\begin{array}{l}0.1047 \\
15\end{array}$ & $\begin{array}{l}119.1379 \\
48785\end{array}$ & 0.103037 & $\begin{array}{l}119.10366 \\
4611\end{array}$ & 0.104240 \\
\hline $\mathrm{CH}_{3} \mathrm{CH}_{2} \mathrm{CHCl}_{2}$ & $\begin{array}{l}- \\
1036.939 \\
6840981\end{array}$ & $\begin{array}{l}0.0869 \\
73\end{array}$ & $\begin{array}{l}- \\
1038.278 \\
05664\end{array}$ & 0.085643 & $\begin{array}{l}- \\
1038.3071 \\
5117\end{array}$ & 0.086489 \\
\hline
\end{tabular}

Figure S1: Calculated geometry for the 1,1-HCl elimination transition state for $\mathrm{CH}_{3} \mathrm{CHCl}_{2}$ at MP2/6-311G(2d,p)

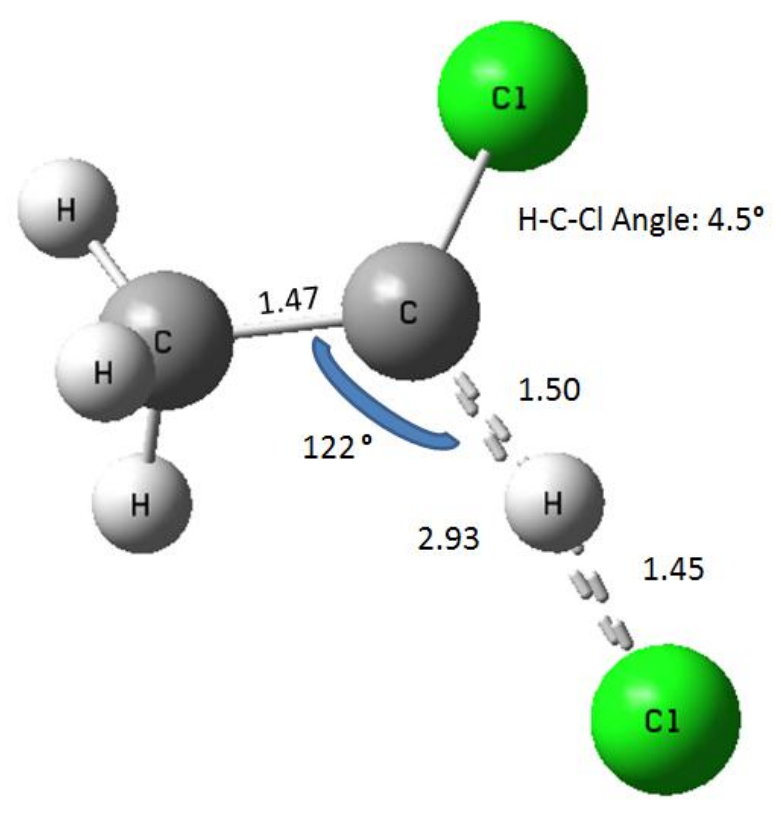




\section{Page S-14 Table S-6 $\mathrm{CD}_{2} \mathrm{ClCHCl}_{2}$ 1,1-HCl Rate constant for}

free internal rotation model at MP2/6-311G(2d,p)

\begin{tabular}{|c|c|c|}
\hline \multirow{2}{*}{$\begin{array}{c}<\mathrm{E}> \\
(\mathbf{k c a l} / \mathbf{m o l})\end{array}$} & \multicolumn{2}{|c|}{ Rate Constant, $\mathrm{k}_{\mathrm{E}}$} \\
\cline { 2 - 3 } & $\mathrm{E}_{0}=70 \mathrm{kcal} / \mathrm{mol}$ & $\mathrm{E}_{0}=68 \mathrm{kcal} / \mathrm{mol}$ \\
\hline $\mathbf{8 2}$ & $0.32 \times 10^{7} \mathrm{~s}^{-1}$ & $1.08 \times 10^{7} \mathrm{~s}^{-1}$ \\
\hline $\mathbf{8 4}$ & $0.80 \times 10^{7} \mathrm{~s}^{-1}$ & $2.44 \times 10^{7} \mathrm{~s}^{-1}$ \\
\hline $\mathbf{8 5}$ & $1.21 \times 10^{7} \mathrm{~s}^{-1}$ & $3.53 \times 10^{7} \mathrm{~s}^{-1}$ \\
\hline $\mathbf{8 6}$ & $1.80 \times 10^{7} \mathrm{~s}^{-1}$ & $5.06 \times 10^{7} \mathrm{~s}^{-1}$ \\
\hline $\mathbf{8 7}$ & $2.60 \times 10^{7} \mathrm{~s}^{-1}$ & $7.04 \times 10^{7} \mathrm{~s}^{-1}$ \\
\hline $\mathbf{8 8}$ & $3.73 \times 10^{7} \mathrm{~s}^{-1}$ & $9.77 \times 10^{7} \mathrm{~s}^{-1}$ \\
\hline $\mathbf{9 0}$ & $7.23 \times 10^{7} \mathrm{~s}^{-1}$ & $17.8 \times 10^{7} \mathrm{~s}^{-1}$ \\
\hline $\mathbf{9 2}$ & $13.2 \times 10^{7} \mathrm{~s}^{-1}$ & $31.8 \times 10^{7} \mathrm{~s}^{-1}$ \\
\hline
\end{tabular}

$\left(I^{\ddagger} / I\right)^{1 / 2}=1.58 \quad S^{\ddagger}=2$

Table S-7 $\mathrm{CD}_{2} \mathrm{ClCHCl}_{2}$ 2,1-DCl (cis and trans) Rate Constant at MP2/6-311G(2d,p)

\begin{tabular}{|c|c|c|}
\hline \multirow{2}{*}{\begin{tabular}{c} 
(kcal/mol) \\
\cline { 2 - 3 }
\end{tabular}} & \multicolumn{2}{|c|}{ Rate Constant, $\mathbf{k}_{\mathrm{E}}$} \\
\cline { 2 - 3 } & $\mathrm{E}_{0}=60 \mathrm{kcal} / \mathrm{mol}$ & $\mathrm{E}_{0}=62 \mathrm{kcal} / \mathrm{mol}$ \\
\hline $\mathbf{8 2}$ & $2.84 \times 10^{7} \mathrm{~s}^{-1}$ & $1.17 \times 10^{7} \mathrm{~s}^{-1}$ \\
\hline $\mathbf{8 4}$ & $4.89 \times 10^{7} \mathrm{~s}^{-1}$ & $2.07 \times 10^{7} \mathrm{~s}^{-1}$ \\
\hline $\mathbf{8 6}$ & $6.30 \times 10^{7} \mathrm{~s}^{-1}$ & $2.72 \times 10^{7} \mathrm{~s}^{-1}$ \\
\hline $\mathbf{8 7}$ & $10.2 \times 10^{7} \mathrm{~s}^{-1}$ & $4.61 \times 10^{7} \mathrm{~s}^{-1}$ \\
\hline $\mathbf{8 8}$ & $12.9 \times 10^{7} \mathrm{~s}^{-1}$ & $5.96 \times 10^{7} \mathrm{~s}^{-1}$ \\
\hline $\mathbf{9 0}$ & $19.8 \times 10^{7} \mathrm{~s}^{-1}$ & $9.50 \times 10^{7} \mathrm{~s}^{-1}$ \\
\hline $\mathbf{9 2}$ & $29.8 \times 10^{7} \mathrm{~s}^{-1}$ & $14.8 \times 10^{7} \mathrm{~s}^{-1}$ \\
\hline
\end{tabular}

$\left(I^{\ddagger} / I\right)^{1 / 2}=1.15 \quad S^{\ddagger}=4$ 


\section{Page S-15}

\section{Table S-8 $\mathrm{CD}_{2} \mathrm{ClCHCl}_{2}$ 1,2-HCl Rate Constant at MP2/6-}

$311 G(2 d, p)$

\begin{tabular}{|c|c|c|}
\hline \multirow{2}{*}{\begin{tabular}{c} 
(kcal/mol) \\
\cline { 3 - 3 }
\end{tabular}} & $\mathrm{E}_{0}=60 \mathrm{kcal} / \mathrm{mol}$ & $\mathrm{E}_{0}=62 \mathrm{kcal} / \mathrm{mol}$ \\
\cline { 2 - 3 } & $0.86 \times 10^{7} \mathrm{~s}^{-1}$ & $0.35 \times 10^{7} \mathrm{~s}^{-1}$ \\
\hline $\mathbf{8 4}$ & $1.50 \times 10^{7} \mathrm{~s}^{-1}$ & $0.63 \times 10^{7} \mathrm{~s}^{-1}$ \\
\hline $\mathbf{8 5}$ & $1.94 \times 10^{7} \mathrm{~s}^{-1}$ & $0.83 \times 10^{7} \mathrm{~s}^{-1}$ \\
\hline $\mathbf{8 6}$ & $2.50 \times 10^{7} \mathrm{~s}^{-1}$ & $1.10 \times 10^{7} \mathrm{~s}^{-1}$ \\
\hline $\mathbf{8 7}$ & $3.18 \times 10^{7} \mathrm{~s}^{-1}$ & $1.42 \times 10^{7} \mathrm{~s}^{-1}$ \\
\hline $\mathbf{8 8}$ & $4.02 \times 10^{7} \mathrm{~s}^{-1}$ & $1.83 \times 10^{7} \mathrm{~s}^{-1}$ \\
\hline $\mathbf{9 0}$ & $6.26 \times 10^{7} \mathrm{~s}^{-1}$ & $2.98 \times 10^{7} \mathrm{~s}^{-1}$ \\
\hline $\mathbf{9 2}$ & $9.46 \times 10^{7} \mathrm{~s}^{-1}$ & $4.65 \times 10^{7} \mathrm{~s}^{-1}$ \\
\hline
\end{tabular}

$\left(I^{\ddagger} / \mathrm{I}\right)^{1 / 2}=1.17 \quad S^{\ddagger}=1$

Ratio of sums of states for the two transition states at $6300 \mathrm{~cm}^{-1}(12$ $\mathrm{kcal} / \mathrm{mol}$ )

$\left(1,1-\mathrm{HCl}^{\ddagger} / 2,1-\mathrm{DCl}^{\ddagger}\right)=\left(22.3^{*} 10^{8}\right) /\left(22.05^{*} 10^{7}\right)=10.6$ 


\section{Page S-16}

\section{Table S-9 $\mathrm{CD}_{3} \mathrm{CD}_{2} \mathrm{CHCl}_{2}$ 1,1- $\mathrm{HCl}$ Rate Constant for free internal rotation model at MP2/6-311G(2d,p)}

\begin{tabular}{|c|c|c|}
\hline \multirow{2}{*}{$\begin{array}{c}\text { (kcal } / \mathrm{mol}) \\
\mathbf{8 5}\end{array}$} & $\mathrm{E}_{0}=70 \mathrm{kcal} / \mathrm{mol}$ & $\mathrm{E}_{0}=67 \mathrm{kcal} / \mathrm{mol}$ \\
\cline { 2 - 3 } & $0.096 \times 10^{6} \mathrm{~s}^{-1}$ & $0.64 \times 10^{6} \mathrm{~s}^{-1}$ \\
\hline $\mathbf{8 7}$ & $0.23 \times 10^{6} \mathrm{~s}^{-1}$ & $1.36 \times 10^{6} \mathrm{~s}^{-1}$ \\
\hline $\mathbf{8 8}$ & $0.34 \times 10^{6} \mathrm{~s}^{-1}$ & $1.93 \times 10^{6} \mathrm{~s}^{-1}$ \\
\hline $\mathbf{8 9}$ & $0.50 \times 10^{6} \mathrm{~s}^{-1}$ & $2.70 \times 10^{6} \mathrm{~s}^{-1}$ \\
\hline $\mathbf{9 1}$ & $1.04 \times 10^{6} \mathrm{~s}^{-1}$ & $5.09 \times 10^{6} \mathrm{~s}^{-1}$ \\
\hline $\mathbf{9 3}$ & $2.04 \times 10^{6} \mathrm{~s}^{-1}$ & $9.40 \times 10^{6} \mathrm{~s}^{-1}$ \\
\hline $\mathbf{9 5}$ & $3.82 \times 10^{6} \mathrm{~s}^{-1}$ & $16.4 \times 10^{6} \mathrm{~s}^{-1}$ \\
\hline
\end{tabular}

$\left(\mathrm{I}^{\ddagger} / \mathrm{I}\right)^{1 / 2}=1.72 \quad \mathrm{~S}^{\ddagger}=2$

Table S-10 $\mathrm{CD}_{3} \mathrm{CD}_{2} \mathrm{CHCl}_{2}$ 2,1-DCl Rate Constant at MP2/6-

\section{$311 G(2 d, p)$}

2,1-DCl Rate constant

\begin{tabular}{|c|c|c|}
\hline \multirow{2}{*}{$(\mathbf{k c a l} / \mathrm{mol})$} & \multicolumn{2}{|c|}{ Rate Constant, $\mathbf{k}_{\mathrm{E}}$} \\
\cline { 2 - 3 } & $\mathrm{E}_{0}=54 \mathrm{kcal} / \mathrm{mol}$ & $\mathrm{E}_{0}=56 \mathrm{kcal} / \mathrm{mol}$ \\
\hline $\mathbf{8 5}$ & $1.71 \times 10^{7} \mathrm{~s}^{-1}$ & $0.70 \times 10^{7} \mathrm{~s}^{-1}$ \\
\hline $\mathbf{8 7}$ & $2.66 \times 10^{7} \mathrm{~s}^{-1}$ & $1.12 \times 10^{7} \mathrm{~s}^{-1}$ \\
\hline $\mathbf{8 8}$ & $3.28 \times 10^{7} \mathrm{~s}^{-1}$ & $1.41 \times 10^{7} \mathrm{~s}^{-1}$ \\
\hline $\mathbf{8 9}$ & $4.02 \times 10^{7} \mathrm{~s}^{-1}$ & $1.80 \times 10^{7} \mathrm{~s}^{-1}$ \\
\hline $\mathbf{9 1}$ & $5.95 \times 10^{7} \mathrm{~s}^{-1}$ & $2.6 \times 10^{7} \mathrm{~s}^{-1}$ \\
\hline $\mathbf{9 3}$ & $8.62 \times 10^{7} \mathrm{~s}^{-1}$ & $4.0 \times 10^{7} \mathrm{~s}^{-1}$ \\
\hline $\mathbf{9 5}$ & $12.3 \times 10^{7} \mathrm{~s}^{-1}$ & $5.8 \times 10^{7} \mathrm{~s}^{-1}$ \\
\hline
\end{tabular}




\section{Page S-17}

$\left(I^{\ddagger} / \mathrm{I}\right)^{1 / 2}=1.28 \quad \mathrm{~S}^{\ddagger}=4$

Ratio of Sum of $\mathrm{P}^{\ddagger}\left(\mathrm{E}-\mathrm{E}_{0}\right)$ of the two transition states at a common energy of $8750 \mathrm{~cm}^{-1} \quad(25 \mathrm{kcal} / \mathrm{mol})$

$\left(1,1-\mathrm{HCl}^{\ddagger} / 2,1-\mathrm{DCl}\right)=\left(2.83^{*} 10^{11}\right) /\left(2.05^{*} 10^{10}\right)=13.8$

\section{Table S-11 $\mathrm{CHCl}_{2} \mathrm{CHCl}_{2}$ 1,1-HCl Rate Constant for Free Rotor Model}

\begin{tabular}{|c|c|c|}
\hline \multirow{2}{*}{\begin{tabular}{c} 
(kcal/mol) \\
\cline { 2 - 3 }
\end{tabular}} & $\mathrm{E}_{0}=70 \mathrm{kcal} / \mathrm{mol}$ & $\mathrm{E}_{0}=68 \mathrm{kcal} / \mathrm{mol}$ \\
\cline { 2 - 3 } & $0.31 \times 10^{6} \mathrm{~s}^{-1}$ & $1.55 \times 10^{6} \mathrm{~s}^{-1}$ \\
\hline $\mathbf{8 0}$ & $1.11 \times 10^{6} \mathrm{~s}^{-1}$ & $4.63 \times 10^{6} \mathrm{~s}^{-1}$ \\
\hline $\mathbf{8 1}$ & $1.96 \times 10^{6} \mathrm{~s}^{-1}$ & $7.60 \times 10^{6} \mathrm{~s}^{-1}$ \\
\hline $\mathbf{8 2}$ & $3.30 \times 10^{6} \mathrm{~s}^{-1}$ & $11.9 \times 10^{6} \mathrm{~s}^{-1}$ \\
\hline $\mathbf{8 3}$ & $5.42 \times 10^{6} \mathrm{~s}^{-1}$ & $18.5 \times 10^{6} \mathrm{~s}^{-1}$ \\
\hline $\mathbf{8 4}$ & $8.71 \times 10^{6} \mathrm{~s}^{-1}$ & $21.2 \times 10^{6} \mathrm{~s}^{-1}$ \\
\hline $\mathbf{8 6}$ & $20.3 \times 10^{6} \mathrm{~s}^{-1}$ & $60.1 \times 10^{6} \mathrm{~s}^{-1}$ \\
\hline $\mathbf{8 8}$ & $44.1 \times 10^{6} \mathrm{~s}^{-1}$ & $127 \times 10^{6} \mathrm{~s}^{-1}$ \\
\hline
\end{tabular}

$\left(I^{\ddagger} / \mathrm{I}\right)^{1 / 2}=1.38 \quad S^{\ddagger}=4$

Table S-12 $\mathrm{CHCl}_{2} \mathrm{CHCl}_{2}$ 2,1-HCl Rate Constant for Free Rotor Model

\begin{tabular}{|c|c|c|}
\hline \multirow{2}{*}{$(\mathbf{k c a l} / \mathbf{m o l})$} & \multicolumn{2}{|c|}{ Rate Constant, $\mathbf{k}_{\mathbf{E}}$} \\
\cline { 2 - 3 } & $\mathrm{E}_{0}=60 \mathrm{kcal} / \mathrm{mol}$ & $\mathrm{E}_{0}=58 \mathrm{kcal} / \mathrm{mol}$ \\
\hline $\mathbf{7 8}$ & $0.62 \times 10^{7} \mathrm{~s}^{-1}$ & $1.75 \times 10^{7} \mathrm{~s}^{-1}$ \\
\hline $\mathbf{8 0}$ & $1.24 \times 10^{7} \mathrm{~s}^{-1}$ & $3.27 \times 10^{7} \mathrm{~s}^{-1}$ \\
\hline $\mathbf{8 1}$ & $1.73 \times 10^{7} \mathrm{~s}^{-1}$ & $4.41 \times 10^{7} \mathrm{~s}^{-1}$ \\
\hline
\end{tabular}




\begin{tabular}{|c|c|c|}
\hline $\mathbf{8 2}$ & $2.35 \times 10^{7} \mathrm{~s}^{-1}$ & $5.84 \times 10^{7} \mathrm{~s}^{-1}$ \\
\hline $\mathbf{8 3}$ & $3.16 \times 10^{7} \mathrm{~s}^{-1}$ & $7.64 \times 10^{7} \mathrm{~s}^{-1}$ \\
\hline $\mathbf{8 4}$ & $4.21 \times 10^{7} \mathrm{~s}^{-1}$ & $9.61 \times 10^{7} \mathrm{~s}^{-1}$ \\
\hline $\mathbf{8 6}$ & $7.20 \times 10^{7} \mathrm{~s}^{-1}$ & $16.2 \times 10^{7} \mathrm{~s}^{-1}$ \\
\hline $\mathbf{8 8}$ & $11.8 \times 10^{7} \mathrm{~s}^{-1}$ & $24.7 \times 10^{7} \mathrm{~s}^{-1}$ \\
\hline
\end{tabular}

$\left(\mathrm{I}^{\ddagger} / \mathrm{I}\right)^{1 / 2}=1.15 \quad \mathrm{~S}^{\ddagger}=4$

Ratio of sums of states for the two transition states at $6300 \mathrm{~cm}^{-1}(12$ $\mathrm{kcal} / \mathrm{mol}$ )

$\left(1,1-\mathrm{HCl}^{\ddagger} / 2,1-\mathrm{HCl}^{\ddagger}\right)=\left(15.7^{*} 10^{8}\right) /\left(2.91 * 10^{8}\right)=5.4$

\section{Page S-18}


Page S-19

Table S-13 Experimental data used to construct Figure 2, D/S vs. 1/P plot. Each reactant was separately measured into a calibrated volume of $13.022 \mathrm{~mL}$ and transferred to the reaction vessel for photolysis. Photolysis times varied depending upon the volume of the reaction vessel.

\begin{tabular}{|c|c|c|c|c|c|c|c|}
\hline $\begin{array}{c}\text { Sample } \\
\text { Name }\end{array}$ & $\begin{array}{c}\text { Reaction } \\
\text { Vessel }\end{array}$ & $\begin{array}{l}C_{2} D_{5} I \\
\text { (torr) }\end{array}$ & $\begin{array}{l}\mathrm{CHCl}_{3} \\
\text { (torr) }\end{array}$ & $\begin{array}{c}\mathrm{CF}_{3} \mathrm{CH}=\mathrm{CH}_{2} \\
\text { (torr) }\end{array}$ & $\begin{array}{l}\text { Photolysis } \\
\text { Time (min) }\end{array}$ & $\begin{array}{c}\text { Pressure } \\
\text { (torr) }\end{array}$ & $1 / P$ \\
\hline acl-i-11d & 9.295 & 5 & 2.008 & 4.003 & 10 & 15.424 & 0.064835 \\
\hline acl-i-12c & 9.295 & 5.006 & 2 & 4.004 & 5 & 15.422 & 0.064841 \\
\hline acl-i-12e & 9.295 & 5 & 2.005 & 3.996 & 5 & 15.41 & 0.064894 \\
\hline acl-i-13a & 9.295 & 1.994 & 1 & 2 & 5 & 6.9954 & 0.14295 \\
\hline$a c l-i-13 b$ & 10.943 & 5 & 2 & 4.001 & 2.5 & 13.089 & 0.076399 \\
\hline acl-i-13c & 9.295 & 4.999 & 1.993 & 8.001 & 5 & 21.001 & 0.047616 \\
\hline$a c l-i-15 d$ & 185.9 & 5.002 & 2.002 & 4 & 2 & 0.77069 & 1.2975 \\
\hline acl-i-15e & 127.53 & 5 & 2 & 3.999 & 0.5 & 1.1229 & 0.89053 \\
\hline acl-i-15f & 109.87 & 5 & 2 & 4.003 & 0.25 & 1.3039 & 0.76693 \\
\hline acl-i-16a & 127.53 & 5 & 2.004 & 3.994 & 0.083333 & 1.1228 & 0.89061 \\
\hline acl-i-16c & 46.902 & 5 & 2.003 & 4 & 0.18333 & 3.0545 & 0.32739 \\
\hline acl-i-16e & 65.467 & 5.0001 & 2 & 4 & 0.16667 & 2.1877 & 0.4571 \\
\hline acl-i-16f & 31.08 & 5 & 2 & 4.001 & 0.25 & 4.6085 & 0.21699 \\
\hline acl-i-17a & 31.08 & 5.001 & 2 & 4 & 0.25 & 4.6085 & 0.21699 \\
\hline$a c l-i-17 b$ & 46.902 & 5 & 2.004 & 3.996 & 0.18333 & 3.0536 & 0.32748 \\
\hline acl-i-17c & 31.08 & 5.001 & 1.998 & 4 & 0.25 & 4.6077 & 0.21703 \\
\hline acl-i-17d & 46.902 & 5 & 2.003 & 4 & 0.18333 & 3.0545 & 0.32739 \\
\hline acl-i-18a & 65.467 & 5.002 & 2 & 3.996 & 0.083333 & 2.1873 & 0.45719 \\
\hline acl-i-18c & 65.467 & 5 & 2.002 & 4.003 & 0.066667 & 2.1887 & 0.4569 \\
\hline acl-i-18d & 127.53 & 5 & 2.002 & 4.001 & 0.066667 & 1.1233 & 0.89021 \\
\hline acl-i-18e & 109.87 & 5.001 & 2.002 & 4.005 & 0.083333 & 1.3045 & 0.76658 \\
\hline acl-i-19e & 185.9 & 5 & 2.002 & 4 & 0.75 & 0.77055 & 1.2978 \\
\hline acl-i-20c & 185.9 & 5.001 & 2 & 3.999 & 1 & 0.77041 & 1.298 \\
\hline
\end{tabular}




\begin{tabular}{|c|c|c|c|c|c|c|}
\hline $\begin{array}{l}(\mathrm{Z}) \\
\mathrm{CD} 3 \mathrm{CD}=\mathrm{CHCl}\end{array}$ & $\begin{array}{l}\text { (E) } \\
\mathrm{CD} 3 \mathrm{CD}=\mathrm{CHCl}\end{array}$ & $\begin{array}{l}(\mathrm{Z}) \\
\mathrm{CD} 3 \mathrm{CD}=\mathrm{CDCl}\end{array}$ & $\begin{array}{l}\text { (E) } \\
C D 3 C D=C D C l\end{array}$ & $\begin{array}{l}\text { CD3CD2CHCl2 } \\
\text { (S) }\end{array}$ & $\begin{array}{l}(\mathrm{Z}) \\
\mathrm{CD} 3 \mathrm{CD}=\mathrm{CHCl} / \mathrm{S}\end{array}$ & $\begin{array}{l}\text { (E) } \\
\mathrm{CD} 3 \mathrm{CD}=\mathrm{CHCl} / \mathrm{S}\end{array}$ \\
\hline $3.34 \mathrm{E}+07$ & $1.41 \mathrm{E}+07$ & $4.96 \mathrm{E}+06$ & $1.70 \mathrm{E}+06$ & $1.18 \mathrm{E}+08$ & 0.28281 & 0.11921 \\
\hline $1.64 \mathrm{E}+07$ & $6.62 \mathrm{E}+06$ & $2.60 \mathrm{E}+06$ & $8.68 \mathrm{E}+05$ & $8.47 E+07$ & 0.19326 & 0.078131 \\
\hline $1.03 \mathrm{E}+07$ & $4.18 \mathrm{E}+06$ & $1.73 E+06$ & $5.58 \mathrm{E}+05$ & $6.37 \mathrm{E}+07$ & 0.16091 & 0.06556 \\
\hline $2.91 \mathrm{E}+07$ & $1.71 \mathrm{E}+07$ & $5.04 \mathrm{E}+06$ & $2.12 \mathrm{E}+06$ & $9.98 \mathrm{E}+07$ & 0.2918 & 0.1717 \\
\hline $8.15 E+06$ & $2.51 E+06$ & $1.23 E+06$ & $3.52 E+05$ & $4.14 \mathrm{E}+07$ & 0.19704 & 0.060697 \\
\hline $7.56 \mathrm{E}+06$ & $3.13 E+06$ & $1.26 \mathrm{E}+06$ & $4.03 E+05$ & $7.30 \mathrm{E}+07$ & 0.10356 & 0.042918 \\
\hline $1.15 \mathrm{E}+08$ & $7.42 \mathrm{E}+07$ & $2.31 \mathrm{E}+07$ & $1.16 \mathrm{E}+07$ & $4.71 E+07$ & 2.441 & 1.5747 \\
\hline $1.02 \mathrm{E}+08$ & $6.46 \mathrm{E}+07$ & $1.83 E+07$ & $8.90 \mathrm{E}+06$ & $6.66 \mathrm{E}+07$ & 1.5249 & 0.97019 \\
\hline $6.46 \mathrm{E}+07$ & $5.45 \mathrm{E}+07$ & $1.34 \mathrm{E}+07$ & $7.01 \mathrm{E}+06$ & $4.25 \mathrm{E}+07$ & 1.5185 & 1.2804 \\
\hline $6.72 \mathrm{E}+07$ & $5.71 \mathrm{E}+07$ & $1.37 \mathrm{E}+07$ & $7.26 \mathrm{E}+06$ & $3.85 E+07$ & 1.7473 & 1.4832 \\
\hline $2.24 \mathrm{E}+07$ & $2.08 \mathrm{E}+07$ & $4.62 E+06$ & $2.44 \mathrm{E}+06$ & $4.72 \mathrm{E}+07$ & 0.47508 & 0.44072 \\
\hline $1.43 \mathrm{E}+07$ & $1.32 \mathrm{E}+07$ & $3.12 \mathrm{E}+06$ & $1.58 \mathrm{E}+06$ & $2.37 \mathrm{E}+07$ & 0.60489 & 0.55926 \\
\hline $9.63 \mathrm{E}+06$ & $8.45 E+06$ & $1.94 \mathrm{E}+06$ & $9.27 \mathrm{E}+05$ & $3.57 \mathrm{E}+07$ & 0.26964 & 0.23656 \\
\hline $1.54 \mathrm{E}+07$ & $1.33 \mathrm{E}+07$ & $3.01 E+06$ & $1.50 \mathrm{E}+06$ & $5.14 \mathrm{E}+07$ & 0.29909 & 0.25834 \\
\hline $3.65 \mathrm{E}+07$ & $3.33 \mathrm{E}+07$ & $7.53 E+06$ & $3.86 \mathrm{E}+06$ & $8.11 \mathrm{E}+07$ & 0.44996 & 0.41062 \\
\hline $2.24 \mathrm{E}+07$ & $1.81 \mathrm{E}+07$ & $4.39 E+06$ & $2.13 E+06$ & $7.04 \mathrm{E}+07$ & 0.31834 & 0.25712 \\
\hline $2.70 \mathrm{E}+07$ & $2.43 \mathrm{E}+07$ & $5.62 E+06$ & $2.95 \mathrm{E}+06$ & $6.02 E+07$ & 0.44774 & 0.40339 \\
\hline $4.50 \mathrm{E}+07$ & $4.09 \mathrm{E}+07$ & $9.43 E+06$ & $4.96 \mathrm{E}+06$ & $5.42 E+07$ & 0.83006 & 0.75525 \\
\hline $2.44 \mathrm{E}+07$ & $2.22 \mathrm{E}+07$ & $5.19 E+06$ & $2.60 \mathrm{E}+06$ & $3.01 \mathrm{E}+07$ & 0.80992 & 0.73827 \\
\hline $6.83 E+07$ & $5.87 \mathrm{E}+07$ & $1.41 \mathrm{E}+07$ & $7.61 \mathrm{E}+06$ & $3.94 \mathrm{E}+07$ & 1.7314 & 1.4883 \\
\hline $2.42 \mathrm{E}+07$ & $2.21 \mathrm{E}+07$ & $5.14 \mathrm{E}+06$ & $2.72 E+06$ & $2.08 \mathrm{E}+07$ & 1.1597 & 1.0582 \\
\hline $9.79 \mathrm{E}+06$ & $5.07 E+06$ & $1.72 E+06$ & $6.71 \mathrm{E}+05$ & $2.79 E+06$ & 3.502 & 1.8141 \\
\hline $1.14 \mathrm{E}+07$ & $6.36 \mathrm{E}+06$ & $2.06 \mathrm{E}+06$ & $8.96 \mathrm{E}+05$ & $3.86 E+06$ & 2.9423 & 1.6491 \\
\hline
\end{tabular}


Page S-21

\begin{tabular}{|c|c|c|c|c|c|}
\hline $\begin{array}{c}(Z) \\
C D 3 C D=C D C l / S \\
\end{array}$ & $\begin{array}{c}(\mathrm{E}) \\
\mathrm{CD} 3 \mathrm{CD}=\mathrm{CDCl} / \mathrm{S}\end{array}$ & $\begin{array}{l}\text { Calibrated (Z) } \\
\mathrm{CD} 3 \mathrm{CD}=\mathrm{CHCl} / \mathrm{S}\end{array}$ & $\begin{array}{l}\text { Calibrated (E) } \\
C D 3 C D=C H C l / S\end{array}$ & $\begin{array}{l}\text { Calibrated }(Z) \\
C D 3 C D=C D C l / S\end{array}$ & $\begin{array}{l}\text { Calibrated (E) } \\
C D 3 C D=C D C l / S\end{array}$ \\
\hline 0.041979 & 0.014375 & 0.15555 & 0.065567 & 0.023089 & 0.007906 \\
\hline 0.030668 & 0.01025 & 0.10629 & 0.042972 & 0.016867 & 0.0056374 \\
\hline 0.027099 & 0.0087551 & 0.0885 & 0.036058 & 0.014905 & 0.0048153 \\
\hline 0.050531 & 0.02123 & 0.16049 & 0.094434 & 0.027792 & 0.011677 \\
\hline 0.029781 & 0.0085123 & 0.10837 & 0.033384 & 0.01638 & 0.0046818 \\
\hline 0.017233 & 0.005517 & 0.056956 & 0.023605 & 0.0094781 & 0.0030343 \\
\hline 0.49052 & 0.24636 & 1.3426 & 0.86607 & 0.26979 & 0.1355 \\
\hline 0.27491 & 0.13379 & 0.83871 & 0.53361 & 0.1512 & 0.073585 \\
\hline 0.31584 & 0.16489 & 0.83515 & 0.70425 & 0.17371 & 0.090688 \\
\hline 0.35682 & 0.18858 & 0.96103 & 0.81576 & 0.19625 & 0.10372 \\
\hline 0.097938 & 0.051831 & 0.2613 & 0.2424 & 0.053866 & 0.028507 \\
\hline 0.13207 & 0.066575 & 0.33269 & 0.30759 & 0.072636 & 0.036616 \\
\hline 0.05425 & 0.025979 & 0.1483 & 0.13011 & 0.029838 & 0.014289 \\
\hline 0.058591 & 0.029202 & 0.1645 & 0.14209 & 0.032225 & 0.016061 \\
\hline 0.092941 & 0.047564 & 0.24748 & 0.22584 & 0.051117 & 0.02616 \\
\hline 0.062345 & 0.030289 & 0.17509 & 0.14142 & 0.03429 & 0.016659 \\
\hline 0.093399 & 0.04905 & 0.24626 & 0.22186 & 0.051369 & 0.026977 \\
\hline 0.17407 & 0.091542 & 0.45653 & 0.41539 & 0.095736 & 0.050348 \\
\hline 0.17235 & 0.086401 & 0.44545 & 0.40605 & 0.094792 & 0.04752 \\
\hline 0.35666 & 0.19296 & 0.95229 & 0.81857 & 0.19617 & 0.10613 \\
\hline 0.24672 & 0.13043 & 0.63781 & 0.582 & 0.13569 & 0.071737 \\
\hline 0.61662 & 0.24 & 1.9261 & 0.99775 & 0.33914 & 0.132 \\
\hline 0.53332 & 0.23218 & 1.6183 & 0.907 & 0.29332 & 0.1277 \\
\hline
\end{tabular}


Page S-22

Table S-14 Experimental data used to construct Figure 3. Each reactant was separately measured into a calibrated volume of $13.022 \mathrm{~mL}$ and transferred to the reaction vessel.

\begin{tabular}{|c|c|c|c|c|c|c|c|}
\hline $\begin{array}{l}\text { Run } \\
\text { Number }\end{array}$ & $\begin{array}{l}\text { Rxn Vessel } \\
(\mathrm{mL})\end{array}$ & $\begin{array}{l}\mathrm{C}_{2} \mathrm{D}_{5} \mathrm{I} \\
\text { (Torr) }\end{array}$ & $\begin{array}{l}\mathrm{CHCl}_{3} \\
\text { (Torr) }\end{array}$ & $\begin{array}{l}\mathrm{CF}_{3} \mathrm{CH}=\mathrm{CH}_{2} \\
\text { (Torr) }\end{array}$ & $\begin{array}{l}\text { Photo } \\
\text { Time(min) }\end{array}$ & $\begin{array}{l}\text { Pressure } \\
\text { (Torr) }\end{array}$ & 1/Press \\
\hline acl-i-11d & 9.295 & 5 & 2.008 & 4.003 & 10 & 15.424 & 0.064835 \\
\hline acl-i-12c & 9.295 & 5.006 & 2 & 4.004 & 5 & 15.422 & 0.064841 \\
\hline acl-i-12e & 9.295 & 5 & 2.005 & 3.996 & 5 & 15.41 & 0.064894 \\
\hline acl-i-13a & 9.295 & 1.994 & 1 & 2 & 5 & 6.9954 & 0.14295 \\
\hline$a c l-i-13 b$ & 10.943 & 5 & 2 & 4.001 & 2.5 & 13.089 & 0.076399 \\
\hline acl-i-13c & 9.295 & 4.999 & 1.993 & 8.001 & 5 & 21.001 & 0.047616 \\
\hline acl-i-15e & 127.53 & 5 & 2 & 3.999 & 0.5 & 1.1229 & 0.89053 \\
\hline acl-i-15f & 109.87 & 5 & 2 & 4.003 & 0.25 & 1.3039 & 0.76693 \\
\hline acl-i-16a & 127.53 & 5 & 2.004 & 3.994 & 0.083333 & 1.1228 & 0.89061 \\
\hline acl-i-16c & 46.902 & 5 & 2.003 & 4 & 0.18333 & 3.0545 & 0.32739 \\
\hline acl-i-16e & 65.467 & 5.0001 & 2 & 4 & 0.16667 & 2.1877 & 0.4571 \\
\hline acl-i-16f & 31.08 & 5 & 2 & 4.001 & 0.25 & 4.6085 & 0.21699 \\
\hline acl-i-17a & 31.08 & 5.001 & 2 & 4 & 0.25 & 4.6085 & 0.21699 \\
\hline acl-i-17b & 46.902 & 5 & 2.004 & 3.996 & 0.18333 & 3.0536 & 0.32748 \\
\hline acl-i-17c & 31.08 & 5.001 & 1.998 & 4 & 0.25 & 4.6077 & 0.21703 \\
\hline acl-i-17d & 46.902 & 5 & 2.003 & 4 & 0.18333 & 3.0545 & 0.32739 \\
\hline acl-i-18a & 65.467 & 5.002 & 2 & 3.996 & 0.083333 & 2.1873 & 0.45719 \\
\hline acl-i-18c & 65.467 & 5 & 2.002 & 4.003 & 0.066667 & 2.1887 & $0.456 \mathrm{~s}$ \\
\hline acl-i-18d & 127.53 & 5 & 2.002 & 4.001 & 0.066667 & 1.1233 & 0.89021 \\
\hline acl-i-18e & 109.87 & 5.001 & 2.002 & 4.005 & 0.083333 & 1.3045 & 0.76658 \\
\hline acl-i-18f & 185.9 & 5.001 & 2 & 3.997 & 0.066667 & 0.77027 & 1.2982 \\
\hline acl-i-19a & 245.29 & 5 & 2 & 3.998 & 0.083333 & 0.58377 & 1.713 \\
\hline acl-i-19b & 245.29 & 5.001 & 2.001 & 3.994 & 0.083333 & 0.58367 & 1.7133 \\
\hline acl-i-19d & 399.03 & 5 & 1.999 & 3.995 & 0.083333 & 0.35872 & 2.7877 \\
\hline acl-i-20a & 399.03 & 5.002 & 1.996 & 4.001 & 0.13333 & 0.35889 & 2.7864 \\
\hline acl-i-20b & 329.03 & 5 & 1.996 & 4 & 0.11667 & 0.43513 & 2.2982 \\
\hline acl-i-20d & 329.03 & 5 & 2 & 4 & 0.11667 & 0.43528 & 2.2974 \\
\hline acl-i-20e & 245.29 & 4.996 & 2 & 3.996 & 0.083333 & 0.58346 & 1.7139 \\
\hline acl-i-20f & 329.03 & 5.004 & 2.002 & 4 & 0.11667 & 0.43552 & 2.2961 \\
\hline acl-i-21a & 399.03 & 5.001 & 2.002 & 4 & 0.13333 & 0.35902 & 2.7854 \\
\hline acl-i-23a & 1056.6 & 5.001 & 1.998 & 4.002 & 0.33333 & 0.13556 & 7.3766 \\
\hline acl-i-23c & 1056.6 & 5.001 & 2.004 & 4 & 0.33333 & 0.13561 & 7.3741 \\
\hline acl-i-23e & 1056.6 & 5 & 1.997 & 3.998 & 0.33333 & 0.13549 & 7.3806 \\
\hline
\end{tabular}




\begin{tabular}{|c|c|c|c|c|c|c|}
\hline $\begin{array}{l}\text { (Z) } \\
\mathrm{CD}_{3} \mathrm{CD}=\mathrm{CHCl}\end{array}$ & $\begin{array}{l}\text { (E) } \\
\mathrm{CD} 3 \mathrm{CD}=\mathrm{CHCl}\end{array}$ & $\begin{array}{l}(\mathrm{Z}) \\
\mathrm{CD}_{3} \mathrm{CD}=\mathrm{CDCl}\end{array}$ & $\begin{array}{l}(\mathrm{E}) \\
\mathrm{CD}_{3} \mathrm{CD}=\mathrm{CDCl}\end{array}$ & cis/trans-1,1 & cis/trans-1,2 & $2,1-\mathrm{DCl} / 1,1-\mathrm{HCl}$ \\
\hline $3.34 \mathrm{E}+07$ & $1.41 \mathrm{E}+07$ & $4.96 \mathrm{E}+06$ & $1.70 \mathrm{E}+06$ & 2.9204 & 2.3723 & 7.1339 \\
\hline $1.64 \mathrm{E}+07$ & $6.62 \mathrm{E}+06$ & $2.60 \mathrm{E}+06$ & $8.68 \mathrm{E}+05$ & 2.9921 & 2.4735 & 6.6326 \\
\hline $1.03 \mathrm{E}+07$ & $4.18 \mathrm{E}+06$ & $1.73 \mathrm{E}+06$ & $5.58 \mathrm{E}+05$ & 3.0953 & 2.4544 & 6.3163 \\
\hline $2.91 \mathrm{E}+07$ & $1.71 \mathrm{E}+07$ & $5.04 \mathrm{E}+06$ & $2.12 \mathrm{E}+06$ & 2.3802 & 1.6995 & 6.4589 \\
\hline $8.15 E+06$ & $2.51 \mathrm{E}+06$ & $1.23 \mathrm{E}+06$ & $3.52 E+05$ & 3.4986 & 2.2462 & 6.7306 \\
\hline $7.56 \mathrm{E}+06$ & $3.13 \mathrm{E}+06$ & $1.26 \mathrm{E}+06$ & $4.03 E+05$ & 3.1236 & 2.4129 & 6.4385 \\
\hline $1.02 \mathrm{E}+08$ & $6.46 \mathrm{E}+07$ & $1.83 \mathrm{E}+07$ & $8.90 \mathrm{E}+06$ & 2.0548 & 1.5718 & 6.105 \\
\hline $6.46 \mathrm{E}+07$ & $5.45 \mathrm{E}+07$ & $1.34 \mathrm{E}+07$ & $7.01 \mathrm{E}+06$ & 1.9155 & 1.1859 & 5.8222 \\
\hline $6.72 \mathrm{E}+07$ & $5.71 \mathrm{E}+07$ & $1.37 \mathrm{E}+07$ & $7.26 \mathrm{E}+06$ & 1.8921 & 1.1781 & 5.9233 \\
\hline $2.24 \mathrm{E}+07$ & $2.08 \mathrm{E}+07$ & $4.62 \mathrm{E}+06$ & $2.44 \mathrm{E}+06$ & 1.8896 & 1.078 & 6.1148 \\
\hline $1.43 \mathrm{E}+07$ & $1.32 \mathrm{E}+07$ & $3.12 \mathrm{E}+06$ & $1.58 \mathrm{E}+06$ & 1.9837 & 1.0816 & 5.8606 \\
\hline $9.63 \mathrm{E}+06$ & $8.45 \mathrm{E}+06$ & $1.94 \mathrm{E}+06$ & $9.27 \mathrm{E}+05$ & 2.0882 & 1.1398 & 6.3095 \\
\hline $1.54 \mathrm{E}+07$ & $1.33 \mathrm{E}+07$ & $3.01 E+06$ & $1.50 \mathrm{E}+06$ & 2.0064 & 1.1577 & 6.3493 \\
\hline $3.65 \mathrm{E}+07$ & $3.33 \mathrm{E}+07$ & $7.53 E+06$ & $3.86 \mathrm{E}+06$ & 1.954 & 1.0958 & 6.1249 \\
\hline $2.24 \mathrm{E}+07$ & $1.81 \mathrm{E}+07$ & $4.39 E+06$ & $2.13 \mathrm{E}+06$ & 2.0583 & 1.2381 & 6.2122 \\
\hline $2.70 \mathrm{E}+07$ & $2.43 \mathrm{E}+07$ & $5.62 \mathrm{E}+06$ & $2.95 \mathrm{E}+06$ & 1.9042 & 1.1099 & 5.975 \\
\hline $4.50 \mathrm{E}+07$ & $4.09 \mathrm{E}+07$ & $9.43 E+06$ & $4.96 \mathrm{E}+06$ & 1.9015 & 1.0991 & 5.9686 \\
\hline $2.44 \mathrm{E}+07$ & $2.22 \mathrm{E}+07$ & $5.19 E+06$ & $2.60 \mathrm{E}+06$ & 1.9948 & 1.097 & 5.9833 \\
\hline $6.83 E+07$ & $5.87 \mathrm{E}+07$ & $1.41 \mathrm{E}+07$ & $7.61 \mathrm{E}+06$ & 1.8484 & 1.1634 & 5.8582 \\
\hline $2.42 \mathrm{E}+07$ & $2.21 \mathrm{E}+07$ & $5.14 \mathrm{E}+06$ & $2.72 E+06$ & 1.8916 & 1.0959 & 5.8806 \\
\hline $4.34 \mathrm{E}+06$ & $3.68 \mathrm{E}+06$ & $9.30 \mathrm{E}+05$ & $4.63 \mathrm{E}+05$ & 2.0075 & 1.1809 & 5.7561 \\
\hline $1.36 \mathrm{E}+07$ & $1.19 \mathrm{E}+07$ & $2.81 \mathrm{E}+06$ & $1.62 \mathrm{E}+06$ & 1.7404 & 1.1408 & 5.7511 \\
\hline $6.55 \mathrm{E}+06$ & $5.23 \mathrm{E}+06$ & $1.32 \mathrm{E}+06$ & $6.70 \mathrm{E}+05$ & 1.9649 & 1.2516 & 5.931 \\
\hline $9.19 \mathrm{E}+06$ & $7.21 \mathrm{E}+06$ & $1.86 \mathrm{E}+06$ & $1.04 \mathrm{E}+06$ & 1.7918 & 1.2749 & 5.6679 \\
\hline $1.00 \mathrm{E}+07$ & $7.75 \mathrm{E}+06$ & $1.99 \mathrm{E}+06$ & $1.06 \mathrm{E}+06$ & 1.8806 & 1.2963 & 5.8462 \\
\hline $2.76 \mathrm{E}+06$ & $2.08 \mathrm{E}+06$ & $5.73 \mathrm{E}+05$ & $2.71 \mathrm{E}+05$ & 2.113 & 1.3229 & 5.7272 \\
\hline $3.89 \mathrm{E}+06$ & $3.24 \mathrm{E}+06$ & $8.14 \mathrm{E}+05$ & $5.07 \mathrm{E}+05$ & 1.6068 & 1.2005 & 5.399 \\
\hline $1.44 \mathrm{E}+07$ & $1.25 \mathrm{E}+07$ & $2.96 \mathrm{E}+06$ & $1.66 \mathrm{E}+06$ & 1.7888 & 1.1546 & 5.8303 \\
\hline $1.32 \mathrm{E}+07$ & $1.07 \mathrm{E}+07$ & $2.68 \mathrm{E}+06$ & $1.44 \mathrm{E}+06$ & 1.8694 & 1.2331 & 5.7868 \\
\hline $1.29 \mathrm{E}+07$ & $9.85 \mathrm{E}+06$ & $2.52 \mathrm{E}+06$ & $1.34 \mathrm{E}+06$ & 1.8884 & 1.3059 & 5.8904 \\
\hline $1.18 \mathrm{E}+07$ & $8.00 \mathrm{E}+06$ & $2.29 \mathrm{E}+06$ & $1.25 \mathrm{E}+06$ & 1.8255 & 1.479 & 5.5993 \\
\hline $1.54 \mathrm{E}+07$ & $1.04 \mathrm{E}+07$ & $3.15 \mathrm{E}+06$ & $1.84 \mathrm{E}+06$ & 1.7168 & 1.4844 & 5.163 \\
\hline $8.66 \mathrm{E}+06$ & $4.48 \mathrm{E}+06$ & $1.81 \mathrm{E}+06$ & $8.79 E+05$ & 2.0635 & 1.9338 & 5.185 \\
\hline
\end{tabular}

\section{Page S-23}




\section{Page S-24}

Table S-15 Experimental data used to construct Figure 4. Each reactant was separately measured into a calibrated volume of 13.022 $\mathrm{mL}$ and transferred to the reaction vessel.

\begin{tabular}{|c|c|c|c|c|c|c|c|c|c|c|c|}
\hline $\begin{array}{c}\text { Sample } \\
\text { ID }\end{array}$ & $\begin{array}{c}\text { Reaction } \\
\text { Vessel mL }\end{array}$ & $\begin{array}{l}\mathrm{CHCl}_{3} \\
\text { (torr) }\end{array}$ & $\begin{array}{c}\mathrm{CF}_{3} \mathrm{CH}=\mathrm{CH}_{2} \\
\text { (torr) }\end{array}$ & $\begin{array}{c}\text { Photolysis } \\
\text { Time }\end{array}$ & $\begin{array}{c}\text { Pressure } \\
\text { (torr) }\end{array}$ & 1/Pressure & $\begin{array}{c}\mathrm{CHCl}=\mathrm{CCl}_{2} \\
\text { (D) }\end{array}$ & $\begin{array}{c}\mathrm{CHCl}_{2} \mathrm{CHCl}_{2} \\
\text { (S) }\end{array}$ & $D / S$ & $\begin{array}{c}\text { Calibration } \\
\text { Factor }\end{array}$ & $\begin{array}{c}\text { Calibrated } \\
\text { D/S }\end{array}$ \\
\hline cas-i57d & 29.08 & 4.001 & 4.002 & 3 minutes & 3.5832 & 0.27908 & 1.39E+07 & $6.23 E+06$ & 2.2255 & 0.3886 & 0.86482 \\
\hline cas-i57e & 29.08 & 3.996 & 4.001 & 3 minutes & 3.5805 & 0.27929 & $8.10 E+06$ & $3.10 E+06$ & 2.6108 & 0.3886 & 1.0146 \\
\hline cas-i58b & 29.08 & 3.996 & 4.009 & 3 minutes & 3.5841 & 0.27901 & $8.95 E+06$ & $3.54 \mathrm{E}+06$ & 2.5255 & 0.3886 & 0.9814 \\
\hline cas-i58e & 17.13 & 3.996 & 4.007 & 3 minutes & 6.0828 & 0.1644 & $5.26 \mathrm{E}+06$ & $3.01 E+06$ & 1.7485 & 0.3886 & 0.67948 \\
\hline cas-i59a & 12.959 & 4 & 4.002 & 3 minutes & 8.0397 & 0.12438 & $2.92 E+06$ & $3.59 E+06$ & 0.81415 & 0.3886 & 0.31638 \\
\hline cas-i59b & 17.13 & 4.004 & 4.002 & 3 minutes & 6.0851 & 0.16434 & $5.81 \mathrm{E}+06$ & $4.12 E+06$ & 1.4118 & 0.3886 & 0.54864 \\
\hline cas-i59d & 6.5095 & 3.998 & 4.004 & 3 minutes & 16.005 & 0.06248 & $6.57 \mathrm{E}+05$ & $3.57 E+06$ & 0.18421 & 0.3886 & 0.071585 \\
\hline cas-i60a & 12.959 & 3.991 & 4.007 & 3 minutes & 8.0356 & 0.12445 & $1.84 \mathrm{E}+06$ & $3.48 \mathrm{E}+06$ & 0.52803 & 0.3886 & 0.20519 \\
\hline cas-i60b & 6.5095 & 4 & 4.003 & 3 minutes & 16.007 & 0.062472 & $3.03 E+05$ & $2.30 E+06$ & 0.13169 & 0.3886 & 0.051174 \\
\hline cas-i60c & 12.959 & 3.999 & 4.001 & 3 minutes & 8.0377 & 0.12441 & $2.87 E+06$ & $3.83 E+06$ & 0.7493 & 0.3886 & 0.29118 \\
\hline cas-i60d & 17.13 & 2 & 6 & 3 minutes & 6.0806 & 0.16446 & $3.78 \mathrm{E}+06$ & $3.68 E+06$ & 1.0268 & 0.3886 & 0.39903 \\
\hline cas-i60e & 29.08 & 1.999 & 6.002 & 3 minutes & 3.5823 & 0.27915 & $7.32 E+06$ & $3.98 \mathrm{E}+06$ & 1.8363 & 0.3886 & 0.7136 \\
\hline acl-i-36d & 6.5095 & 4.012 & 3.998 & 7 minutes & 16.021 & 0.062417 & 94273 & $3.81 E+05$ & 0.24719 & 0.3886 & 0.09606 \\
\hline
\end{tabular}

